\title{
Endoscopic treatment of spontaneous, incomplete esophageal rupture in a patient with "crackleware esophagus"
}

A 51-year-old woman presented with acute epigastric pain after ingestion of a rice meal. The patient's history was unremarkable apart from a long-term habit of ingestion of hot peppermint candies and nicotine misuse (20 cigarettes per day). Examination of the oral cavity revealed patchy leukoplakia. Laboratory tests were unremarkable apart from positive antinuclear antibody (ANA) $(1: 320)$ and positive anti-dsDNA antibodies. Gastroscopy disclosed a $6 \mathrm{~cm}$ long, incomplete rupture extending into the muscularis propria of the middle third of the esophagus ( $\bullet$ Fig. 1 a). The surrounding mucosa appeared thickened and showed areas of diffuse keratinization, thus acquiring a "crackleware" appearance. On histological examination, extensive hyperkeratosis was noted ( Fig.1 b). Biopsy specimens taken from the gastroesophageal junction revealed mild reflux disease. Further investigations with respect to esophageal involvement in collagenosisassociated motility disorders (including oesophageal manometry), however, were negative.

Endoscopic treatment was carried out, sealing the rupture by continuous metal clipping ( $\bullet$ Fig. 1 c). The patient was discharged with prescriptions for proton pump inhibitors and antibiotics, and instructions to avoid nicotine and spicy and hot foods. On regular follow-up, it was seen that the treatment was successful with good healing, without visible metal clips or functional impairment (॰ Fig. $1 \mathrm{~d}$ ).

Diffuse hyper-/parakeratosis of the esophagus is an uncommon finding. Only three cases have been reported, two of which had squamous cell carcinoma in the vicinity of the parakeratosis [1-3]. The etiology of this peculiar finding is unclear. The differential diagnosis includes rare inherited keratinization defects, such as tylosis and mucosal hyperkeratosis syndromes,
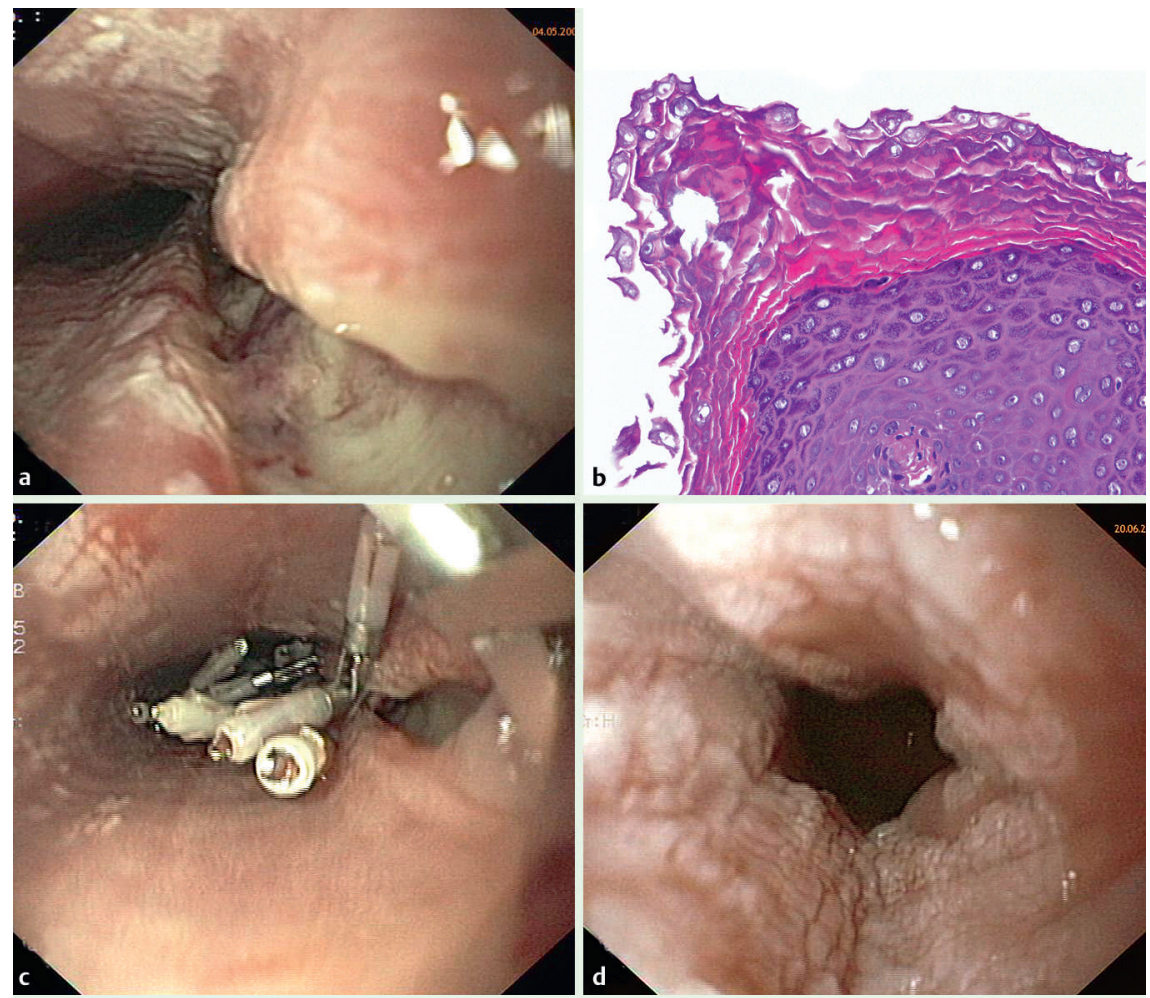

Fig. 1 a Spontaneous, incomplete rupture extending into the muscularis propria of the middle third of the esophagus. $\mathbf{b}$ Extensive hyperkeratosis (magnification $\times 100$ ). $\mathbf{c}$ Endoscopic treatment with continuous metal clipping. $\mathbf{d}$ Follow-up endoscopy showing good healing without visible metal clips or functional impairment.

as well as lichen planus, which commonly affects the proximal esophagus $[4,5]$.

Apart from habitual peppermint candy ingestion and nicotine misuse, the patient's history was unremarkable with respect to potential exogenous toxins. Positive ANA and anti-dsDNA antibodies, however, may indicate a pathogenetic link to autoimmune disease. As shown in our case, diffuse hyper-/parakeratosis may be associated with increased fragility of the esophageal wall, leading to spontaneous rupture. This situation, in addition to the increased cancer risk, should be included in the list of possible complications.
Endoscopy_UCTN_Code_CCL_1AB_2AC_3AH

E. M. Kieninger ${ }^{1}$, F. Siebert ${ }^{2}$, C. Langner ${ }^{1}$ ${ }^{1}$ Institute of Pathology, Medical University of Graz, Austria

2 Department of Internal Medicine, Hospital of Barmherzige Brüder, St. Veit/Glan, Austria 


\section{References}

1 Nakanishi Y, Ochiai A, Shimoda T et al. Epidermization in the esophageal mucosa: unusual epithelial changes clearly detected by Lugol's staining. Am J Surg Pathol 1997; 21: $605-609$

2 Westerterp M, Busch OR, Bergman JJ et al. A "crackleware" oesophagus. J Clin Pathol 2005; 58: 1325 - 1327

3 Tu CH, Tai CM, Chang CY et al. Diffuse esophageal parakeratosis. Endoscopy 2007; 39 (Suppl 1): E119-E120
4 Ashworth MT, Nash JR, Ellis A et al. Abnormalities of differentiation and maturation in the oesophageal squamous epithelium of patients with tylosis: morphological features. Histopathology 1991; 19: 303-310

5 Chandan VS, Murray JA, Abraham SC. Esophageal lichen planus. Arch Pathol Lab Med 2008; 132: 1026 - 1029

Bibliography

DOI $10.1055 / \mathrm{s}-0029-1214658$

Endoscopy 2009; 41: E123-E124

(c) Georg Thieme Verlag KG Stuttgart · New York . ISSN 0013-726X
Corresponding author

\section{Langner, MD}

Institute of Pathology

Medical University of Graz

Auenbruggerplatz 25

A-8036 Graz

Austria

Fax: +43-316-3853432

cord.langner@medunigraz.at 